Opinion

\title{
Artificial evaporation changes the climate
}

\section{Opinion}

The decrease in ice at the poles of the Earth and in the mountains is not the result of warming, but the result of changes in atmospheric phenomena.

Mankind annually alienates from nature all new and new lands: plowing it under crops, pouring all the reservoirs of hydropower plants, concreting and asphalt with new cities and roads. All water evaporated from such territories, at best, does not change its structure. As they came with precipitation, they returned back to the atmosphere. At worst, they are impregnated and combined with chemical elements, which then, it happens that, with a strong concentration, they fall acid rain. Evaporations from artificial areas are supplemented by evaporation and from technological processes of industrial and municipal production. At their core, these fumes are alien to nature. Before the advent of man, such fumes did not exist. You can call them artificial fumes. Nature did not expect such a massive evaporation from washing and drying clothes, dishes, asphalt, $\mathrm{m}$ of all that is created by man.

Historically, the interaction of the plant and animal worlds in a unified symbiosis with atmospheric phenomena created a cycle of substances and water in nature. For millions of years, the volumes and frequency of precipitation, their places of precipitation, regularity have stabilized. The result was the formation of various arid zones: steppes, deserts, forests, tropics - the whole palette of geographic zones, including the comfortable climate in the inhabited man and areas. Polar and mountain glaciers too.

As industrialization develops, artificial evaporation increases in volumes and speeds. The quality of evaporation is a little-studied direction of science, but, in all likelihood, it also has some effect on the "heavenly kitchen." Evaporations from drying asphalt and from the plant or from our breathing cannot be the same.

A promoted official hypothesis based on carbon dioxide emissions distracts the world community from the true cause of climate change and leads the world to a global catastrophe. If during the year 4 billion tons of carbon dioxide is released into the atmosphere, then the water of artificial evaporation alone is 2,200 billion tons.
Volume 3 Issue 6 - 2018

\author{
Halidullin $\mathrm{O}$ \\ Kazakh National University, Russia
}

Correspondence: Halidullin O, Ecology Professor, Academician of the Russian Federation LAN, Kazakh National University, Russia,Tel 87770550099, Email71II5215@mail.ru

Received: October 04, 2018 | Published: November 16, 2018

"Heavenly kitchen" has broken, due to changes in its components. Distribution mechanisms, schedules, dosing of precipitation by zones of the earth's surface have disappeared. In some places, devastating floods, in others - drought and fires.

The mechanism of long-term accumulation of water in glaciers also broke down. The growth of glaciers stopped. Without reaching the poles and mountain zones of water, fall outside the specified ranges. Therefore, the level of the oceans is growing. And this growth increases in some progression.

Is there salvation? It is necessary to return all taken away to nature: land and water.

It is urgent to prove this hypothesis and develop a new concept of saving life on the planet. It should be based on total saving of water in production and everyday life by every enterprise, every state, every person. It is not easy to make rules about forgotten tap and reduce water consumption. It is necessary to radically reconsider all actions related to water. Water must make a qualitative transition from the functions of the working fluid body, the reagent to the living partner. The main purpose of the water is its drinking, consumption. Everything else must be reduced.

\section{Acknowledgements}

None.

\section{Conflict of interest}

Author declares that there is no conflict of interest. 